\title{
Effect of transcutaneous electrical stimulation on morphological characteristics of women with stress urinary incontinence
}

Ji-hyun Kim

Yonsei University

Eun Young Park

Yonsei University College of Medicine

Oh-yun Kwon

Yonsei University

Ui-jae Hwang

Yonsei University

\section{Su-jin Kim}

Yonsei University College of Medicine

\section{Hyeo-bin Yoon}

Yonsei University

Hyeseon Jeon ( $\nabla$ hyeseonj@yonsei.ac.kr)

Yonsei University - Wonju Campus https://orcid.org/0000-0003-3986-2030

\section{Research article}

Keywords: Bladder neck hypermobility, Stress urinary incontinence, Pelvic floor electrical stimulator, Pelvic floor muscle, Transcutaneous electrical stimulation, Ultrasonography

Posted Date: June 17th, 2020

DOI: https://doi.org/10.21203/rs.3.rs-30006/v1

License: (a) (1) This work is licensed under a Creative Commons Attribution 4.0 International License. Read Full License 


\section{Abstract}

Background: Stress urinary incontinence is an involuntary leakage of urine due to a weak pelvic floor and weak sphincter when the intra-abdominal pressure increases. Its symptoms are known to improve upon electrical stimulation of the pelvic floor. This study aimed to determine the effects of transcutaneous electrical stimulation on ultrasonography variables, such as the bladder neck position (BNP), length of the urethra (LU), funneling index (FI), and posterior (PRT) and anterior rhabdosphincter thickness (ART), and the Incontinence Quality of Life scores. It also investigated the association between the relative changes in the two.

Methods: Twenty-one patients with stress urinary continence were included and subjected to transcutaneous electrical stimulation for eight weeks. Ultrasonography was used to measure the BNP, LU, $\mathrm{FI}, \mathrm{ART}$, and PRT. Data were analyzed at rest and during the Valsalva maneuver and the difference $(\triangle)$ between them was evaluated.

Results: The BNP during the Valsalva maneuver and the $\triangle B N P$ decreased significantly. The LU during the Valsalva maneuver increased after eight weeks. The FI during the Valsalva maneuver and the $\triangle \mathrm{FI}$ changed significantly. The ART and PRT and the total quality of life score significantly increased after eight weeks $(p<.05)$. The LU during the Valsalva maneuver positively correlated with the total quality of life $(r=0.630 ; p=.002)$, psychosocial impact subscale $(r=0.705 ; p=.000)$, and social embarrassment subscale $(r=0.488 ; p=.025)$ scores. The correlations between the PRT and the avoidance and limiting behavior subscale score $(r=0.624 ; p=.002)$ and between the $\triangle F I$ and the social embarrassment subscale score $(r=-0.515 ; p=.0 .20)$ were significant.

Conclusions: Transcutaneous electrical stimulation can improve the BNP, ART, and PRT, along with the subjective indicators, in women with stress urinary continence. Improving stress urinary continence symptoms can ameliorate women's social and psychological self-esteem.

\section{Background}

The International Continence Society defines stress urinary incontinence (SUI) as an involuntary loss of urine when the intra-abdominal pressure increases due to sneezing, coughing, or physical exertion $[1,2]$. A possible cause of SUI is bladder neck hypermobility due to pelvic floor muscle (PFM) weakness [3]. SUI affects the patient's social, physical, and psychological well-being and his/her quality of life [4, 5]. However, people with SUI often hesitate to disclose their symptoms. Various noninvasive or conservative interventions, such as medications, pelvic floor muscle training (PFMT), and pelvic floor electrical stimulation (PFES), are available for managing SUI. According to a previous study, noninvasive treatment results in a greater improvement of mild-to-moderate SUI [6].

The PFM, which supports the pelvic viscera and controls the pelvic outlets, is an active contributor to the continence mechanism [7]. During sneezing, coughing, or physical exertion, increases in the intraabdominal pressure are transmitted to the urethra to reinforce its closure only if the urethra remains in 
position between the anterior vaginal wall or the pubic symphysis and the PFM [8]. Assessment of bladder neck hypermobility has revealed that during functional tasks, patients with SUl show a larger excursion of the urethral angle than their continent counterparts $[9,10]$. Bladder neck hypermobility and reduced proximal urethral closure are associated with bladder neck funneling in SUI [11, 12]. Several studies have evaluated bladder neck hypermobility parameters, such as the bladder neck position (BNP), which are associated with the PFM functions $[13,14]$. When the BNP is above the pelvic floor, the pressure transmitted to the bladder is equally transmitted to the urethra, which simultaneously increases the urethral closure pressure. If the BNP is lower than the pelvic floor, a greater pressure is transmitted to the bladder than to the urethra, which threatens the urethral closure and continence status. Due to bladder neck descent, the length of the urethra (LU) is shorter in incontinent women than in continent women [15]. Additionally, the volume of the external sphincter appears smaller in SUI women than in continent women on ultra-sonography [16].

PFES is frequently used in the conservative treatment of patients with SUI. It may lead to nerve regrowth and strengthening of the external sphincter, thereby increasing bladder outlet resistance over time $[17,18]$. It also assists women who face difficulty in identifying and feeling the PFM and also allows an electrically-induced contraction of a weak PFM [19]. Transcutaneous electrical stimulation (TES) and intravaginal electrical stimulation are the two common PFES types for SUI and the effectiveness of each has been proven clinically $[19,20]$. In the intravaginal type, electrodes are inserted into the vagina; therefore, this type could be potentially limited by pain, intolerance to high stimulation intensity, discomfort associated with electrode insertion, difficulty in hygiene management, risk of infections, and/or bleeding. In contrast, the TES is more convenient and acceptable to the user and safer with respect to infections. Recently, the Easy-K (Alphamedic Co., Ltd., Daegu, Korea) device was designed for contracting the PFM by electrical stimulation using cutaneous perivaginal electrodes in the sitting position. Previous studies suggest that TES is effective in treating urinary incontinence and can be performed using cutaneous electrodes in the perivaginal region $[21,22]$.

Thus, the present study aimed to demonstrate the effect of TES on BNP, LU, bladder neck funneling, urethral external sphincter hypertrophy, and quality of life. It also aimed to determine the association between the relative changes in the total and subscale scores of the Incontinence Quality of Life (I-QOL) questionnaire and the relative change in the ultra-sonography variables in women with SUI. The current study hypothesized that the bladder neck hypermobility would improve and the I-QOL scores would be increase after eight weeks of TES.

\section{Methods}

\section{Subjects and design}

The G*power software version 3.1.2 (Franz Faul, University of Kiel, Kiel, Germany) was used for power analysis based on the results of a pilot study on five subjects. Sample size calculation was performed with a power of 0.80 , alpha level of 0.05 , and effect size of 0.95 ; the necessary sample size was found to 
be 10 subjects. A total of 21 women who were diagnosed by gynecologist with grade 1 or grade 2 SUI according to the Stamey's grading system (Table 1) were recruited in this study through a social networking site. Their mean age was 51.3 years and their mean body mass index was $24.12 \mathrm{k} \mathrm{kg} / \mathrm{m}^{2}$. Other demographic characteristic of the subjects is summarized in the Table 2. The exclusion criteria included pregnancy and a history of urogenital surgery such as urinary incontinence surgery, colporrhaphy, and hysterectomy. All subjects read an explanation about the experimental procedures and then signed an informed consent form. This study was approved by the Yonsei University Wonju Institutional Review Board (approval number: 1041849-201808-BM-077-02).

This study was conducted on a single group using a pre- and post- design over an eight-week period. All subjects were assessed twice at baseline and eight weeks after the initiation of the intervention. During the treatment, they were asked to write a daily report on the usage of TES; a researcher checked the subjects' compliance daily through this report and text messages.

\section{Transcutaneous electrical stimulation}

The Easy- $\mathrm{K}$ is a TES device that uses cutaneous electrodes in the perivaginal region for stimulating the PFMs and the surrounding structures (Figure 1); the shape and position of the electrodes are such that the electrodes come in contact with the entire vulva while the user is sitting on the stimulator (Figure 2). A layer of wet tissue is used as a conducting material between the skin and the electrodes. Before using the device, we cleaned the vulva and laid wet tissues on the electrode. This generated an electrical stimulus that directly contacted the perivaginal regions and stimulated the PFM extensively while the subject was sitting on the Easy-K. The device was placed on the toilet seat and the subjects were instructed to sit on the device as the cutaneous electrodes came in contact with the perivaginal regions. Thereafter, the physical therapist set the stimulation amplitude to evoke perceivable and comfortable stimulation levels. The Easy-K delivered biphasic, asymmetric impulses of 21-27 Hz and provided controlling sessions for pulse (5 seconds) and resting (4 seconds) durations. The subjects were instructed to use the device once a day in a 20-minute session for five-to-six days a week over a period of eight weeks. Furthermore, they underwent an Easy-K session with possible increases in the stimulation amplitude (as tolerated by the patients).

\section{Outcome measures}

The subjects were asked not to urinate for three to four hours prior to the examination. The objective variables including the BNP, LU, funneling index (FI), and rhabdosphincter thickness (RT) were measured using an ultrasound scanner (ALOKA, SSD-a10, JAPAN) with a 5.0 Mhz vaginal-type transducer. The subjects were examined in the lithotomy position by a skilled gynecologist. Their morphological characteristics were evaluated at rest and during the Valsalva maneuver. These characteristics were analyzed on frozen images. The differences in the values of these variables between resting and during the Valsalva maneuver were calculated and statistically analyzed; these differences were denoted by delta $(\triangle)$. The BNP $\left(^{\circ}\right)$ was quantified by calculating the pubourethral angle between two axes: The $x$-axis 
was defined by a line drawn from the lower margin of the pubic symphysis to the bladder neck, while the $y$-axis was defined by the midline of the pubic symphysis (Figure 3) [23]. The LU (mm) is the distance from the bladder neck to the lower margin of the pubic symphysis; the longer this distance, the higher is the position of the bladder. The $\mathrm{Fl}$ is measured by multiplying the width $(\mathrm{mm})$ of the bladder neck by its height $(\mathrm{mm})$ [24]. The rhabdosphincters, a part of the external urethral sphincter, are located in the middle of the urethra [16]. The RT $(\mathrm{mm})$ was measured at a distance of $5 \mathrm{~mm}$ from the bladder neck point towards the bladder in the sagittal plane [25]; the anterior RT (ART) and the posterior RT (PRT) are measured from the anterior and posterior walls of the urethra, respectively.

The subjective variables were self-reported using the Korean version of the I-QOL questionnaire. The IQOL is used to assess the incontinence-specific quality of life. It is in the form of a Likert-type response scale and contains 22 items. The subjects assessed themselves using the 5-point scale questions on daily life related to SUI and scored themselves as 1 (extreme), 2 (quite a bit), 3 (moderate), 4 (a little), or 5 (not at all). The total sums of these scores were documented for analysis. There are three subscales: the avoidance and limiting behavioral (AL), psychosocial impacts (PI), and social embarrassment (SE) subscales. A higher total score means a higher self-satisfaction and better SUI-related quality of life.

\section{Statistical analysis}

The data were analyzed using SPSS for Windows version 24.0 (SPSS Inc., Chicago, IL, USA). The Kolmogorov-Smirnov test was performed to confirm the normal distribution of the data. The paired $t$-test was used to analyze the BNP, LU, FI, ART, and PRT in each condition and the I-QOL scores before and after the intervention. Pearson's correlation was used to determine the association between the relative changes in the total and subscale scores of the I-QOL and the ultrasonographic variables in the women with SUI. The significance level was set at a p-value of .05.

\section{Results}

Table 3 presents the ultrasonographic findings. The BNP during the Valsalva maneuver and the difference values representing bladder neck hypermobility significantly decreased after eight weeks of intervention $(p<.05)$. The LU significantly increased after eight weeks only during the Valsalva maneuver $(p<.05)$. While the $\mathrm{FI}$ during the Valsalva maneuver and the $\triangle \mathrm{FI}$ decreased significantly $(\mathrm{p}<.05)$, the $\mathrm{FI}$ at rest did not improve significantly. The ART and PRT, which are the indicators of external sphincter hypertrophy and were measured both at rest and during the Valsalva maneuver, significantly increased post intervention $(p<.05)$; the $\triangle$ ART also increased significantly $(p<.05)$.

The total I-QOL score increased from 64.81 to 71.86 after intervention $(p<.05)$. Among the three subscale scores, the AL and PI sub-scores significantly improved ( $p<.05)$. Although the SE sub-score did not improve significantly ( $p>.05$ ), it steadily increased from 14.33 to 14.9 over the eight-week treatment period (Table 4). 
Table 5 indicates the Pearson's correlation between the I-QOL sub-scores and the BNP, LU, FI, ART, and PRT. Significant positive correlations were observed between the total I-QOL score and the LU during the Valsalva maneuver, between the AL sub-score and the PRT at rest and during the Valsalva maneuver, between the PI sub-score and the LU during the Valsalva maneuver, and between the SE sub-score and the $\triangle \mathrm{FI}$ and LU during the Valsalva maneuver.

\section{Discussion}

The present study aimed to demonstrate the effects of TES on bladder neck hypermobility, morphological characteristics, and psychological satisfaction in women with SUI. To our knowledge, this study is the first to evaluate the BNP, LU, FI, ART, and PRT using ultra-sonography after eight weeks of TES in women with SUI. Although previous studies have suggested that PFMT may decrease bladder neck hypermobility in SUI [3,26], no studies have investigated the improvement of bladder neck hypermobility after TES of the PFM. The main findings of the present study indicate that TES could improve not only the bladder neck hypermobility, but also the subjective symptoms of SUI. Therefore, TES is beneficial for improving the subjective symptoms in patients with SUI by improving the bladder neck hypermobility, which is the fundamental mechanism of SUI [3].

The stimulus is transmitted by the afferent pudendal nerve to the efferent pudendal nerve and the hypogastric nerve induces the contraction of the periurethral muscle and the PFM [27]. In patients with $\mathrm{SUI}$, this pudendal nerve conduction is disturbed, which along with sphincter and PFM weakness, delays the reflexive response of the PFM [17]. The electrodes of the TES device are positioned to fit the dermatome of the pudendal nerve and the base of the pubococcygeus and iliococcygeus muscles, which belong to the PFM. Because TES directly stimulates the muscle, it may be effective in not only strengthening the muscles, but also in imparting the ability of identifying and recognizing the location and contraction of the PFM to women who cannot contract the muscle voluntarily [19]. These effects can restore the normal neuromuscular activities [19]. Restoring neuromuscular control and PFM function increases the bladder neck stability and urethral closure $[17,18]$. The TES-induced muscle contraction provides an involuntary exercise for improving the urethral closure mechanism [19]. Hahn et al. reported that though TES and PFMT have similar positive effects [28], achieving accurate PFMT effects is difficult due to an insufficient PFM force and the poor perception of PFM contraction in patients with SUI. Therefore, electrical stimulation could help the perception of PFM contraction by direct stimulation [28, 29].

We found moderate to high correlations between the I-QOL scores and the variables of ultra-sonography. There was a positive correlation between LU under Valsalva and the total score $(r=0.630 ; p=.002), \mathrm{PI}$ $(r=0.705 ; p=.000)$, and SE $(r=0.488 ; p=.025)$. This indicates that the total score and the PI and SE subscores of I-QOL increase as the LU during the Valsalva maneuver increases. Incontinent women have a shorter LU as compared to continent women, especially during the Valsalva maneuver, because the bladder neck funneling increases and the LU decreases [25]. Furthermore, Kim et al reported that the LU of SUI women increased, with an improvement in the SUI symptoms, after eight weeks of TES [25]. In this 
study, we identified that an increase in the LU is highly correlated with the SUI women's subjective symptoms. The increase in LU after TES may be related to the reduction of subjective symptoms because a functional LU was obtained. We also found a positive correlation between the PRT and AL ( $r=0.624$; $p=.002$ ). The rhabdosphincters are directly connected to the PFM and play an important role as urine flow regulators. Therefore, TES could indirectly affect the PRT by improving the AL score. Finally, we observed a correlation between $\triangle \mathrm{FI}$ and $\mathrm{SE}(r=-0.515 ; p=.0 .20)$. The higher a woman's $\mathrm{Fl}$, the higher the pressure of the urine from the bladder to the urethra. This finding could indicate an improvement in the urethral pressure against the load after eight weeks of TES. In conclusion, ultra-sonography variables could serve as important clinical indicators for determining the most reasonable mechanism behind the improvement of SUl symptoms after TES

The findings of our study can provide evidence that TES improves the ability of maintaining urinary continence through the development of a strong and rapid voluntary control of the PFM and sphincters in women with SUI when their intra-abdominal pressure increases during sneezing, coughing, or physical exertion. TES is beneficial in improving the stability of the PFM and sphincter support systems by providing an adequate resistive force against increased intra-abdominal pressure [30]. We believe that in this experiment, the anticipatory and reactive abilities of individuals with SUI may have been greatly affected by the improvement of the AL and PI sub-scores of I-QOL. However, this study has certain limitations. Firstly, it is difficult to generalize our result because this experiment included a relatively small sample size and did not include a control group. Secondly, we did not measure parameters such as electromyography data, strength, power, and endurance of the PFM. Further research with a larger sample size and a control group is necessary. It is essential to identify improvements in the neuromuscular function of the PFM through electromyography in women with SUI.

\section{Conclusions}

The present study identified that an eight-week course of TES affected the BNP, LU, FI, ART, PRT, and IQOL scores of SUI women. TES can be beneficial as a conservative method for improving bladder neck hypermobility, urethral sphincter thickness, and subjective symptoms in women with SUI. Improving the SUl symptoms can ameliorate the women's self-esteem in social and psychological aspects. The study findings may be useful for developing guidelines for SUI treatment.

\section{Abbreviations}

ART

Anterior rhabdosphincter thickness

AL

Avoidance and limiting behavioral

BNP

Bladder neck position

$\mathrm{FI}$ 
Funneling index

I-QOL

Incontinence Quality of Life

LU

Length of the urethra

PFES

Pelvic floor electrical stimulation

PFM

Pelvic floor muscles

PFMT

Pelvic floor muscles training

$\mathrm{PI}$

Psychosocial impacts

PRT

Posterior rhabdosphincter thickness

RT

Rhabdosphincter thickness

SE

Social embarrassment

SUI

Stress urinary incontinence

TES

Transcutaneous electrical stimulation

\section{Declarations}

\section{Ethics approval and consent to participate}

All subjects provided written consent to participate. This study was approved by the Yonsei University Wonju Institutional Review Board (approval number: 1041849-201808-BM-077-02).

\section{Consent for publication}

The present study obtained publishing consent from participants.

\section{Availability of data and materials}

The datasets used and/or analyzed during the present study are available from the corresponding author on reasonable request.

\section{Competing interests}

The authors declare that they have no competing interests. 


\section{Funding}

The authors received financial and administrative support from the Yonsei University Research Fund (grant numbers 2018-51-0251). The funders had no role in the study design, data collection and analysis, decision to publish or preparation of the manuscript.

\section{Authors' contributions}

OYK, HSJ and UJH contributed to the design and conception of the study. HBY was responsible for recruitment, enrollment, and follow-up of patients. EYP and SJK contributed to the acquisition of data and interpretation of the data. UJH and JHK performed the statistical analysis. JHK and HSJ wrote the first draft of the manuscript. UJH and OYK substantively revised it. All authors read and approved the final manuscript.

\section{Acknowledgements}

We would like to thank the Yonsei University and the participants for their support.

\section{References}

1. Abrams P, Cardozo L, Fall M, Griffiths D, Rosier P, Ulmsten U, et al. The standardisation of terminology of lower urinary tract function: report from the Standardisation Sub-committee of the International Continence Society. Neurourol Urodyn. 2002;21:167-78.

2. Buckley BS, Lapitan M. Prevalence of urinary incontinence in men, women, and children-current evidence: findings of the Fourth International Consultation on Incontinence. Urology. 2010;76:265-70.

3. Hung HC, Hsiao SM, Chih SY, Lin HH, Tsauo JY. Effect of pelvic-floor muscle strengthening on bladder neck mobility: a clinical trial. Phys Ther. 2011;91:1030-8.

4. Jha S, Walters SJ, Bortolami O, Dixon S, Alshreef A. Impact of pelvic floor muscle training on sexual function of women with urinary incontinence and a comparison of electrical stimulation versus standard treatment (IPSU trial): a randomised controlled trial. Physiotherapy. 2018;104:91-7.

5. Wyman JF, Harkins SW, Choi SC, Taylor JR, Fantl J. Psychosocial impact of urinary incontinence in women. Obstet Gynecol. 1987;70:378-81.

6. Syan R, Brucker BM. Guideline of guidelines: urinary incontinence. BJU Int. 2016;117:20-33.

7. Sapsford R. The pelvic floor: a clinical model for function and rehabilitation. Physiotherapy. 2001;87:620-30.

8. DeLancey J, Gosling J, Creed K, Dixon J, Delmas V, Landon D, et al. Gross anatomy and cell biology of the lower urinary tract. In: Abrams P, Cardozo L, Khoury S, Wein A, editors. Incontinence. Plymouth, UK: Health Publication; 2002. p. 19-82.

9. Cassadó J, Pessarrodona A, Tulleuda R, Cabero L, Valls M, Quintana S, et al. Introital ultrasonography: a comparison of women with stress incontinence due to urethral hypermobility and 
continent women. BJU Int. 2006;98:822-8.

10. Shek KL, Dietz HP. The urethral motion profile: a novel method to evaluate urethral support and mobility. Aust N Z J Obstet Gynaecol. 2008;48:337-42.

11. Kuo HC. Transrectal sonography of the female urethra in incontinence and frequency-urgency syndrome. J Ultrasound Med. 1996;15:363-70.

12. Dietz $\mathrm{H}$, Clarke $B$. The urethral pressure profile and ultrasound imaging of the lower urinary tract. Int Urogynecol J. 2001;12:38-41.

13. Thompson JA, O’Sullivan PB, Briffa NK, Neumann P. Comparison of transperineal and transabdominal ultrasound in the assessment of voluntary pelvic floor muscle contractions and functional manoeuvres in continent and incontinent women. Int Urogynecol J. 2007;18:779-86.

14. Miller JM, Perucchini D, Carchidi LT, DeLancey JO, Ashton-Miller J. Pelvic floor muscle contraction during a cough and decreased vesical neck mobility. Obstet Gynecol. 2001;97:255-60.

15. Rud T. Urethral pressure profile in continent women from childhood to old age. Acta Obstet Gynecol Scand. 1980;59:331-5.

16. Klauser A, Frauscher F, Strasser H, Helweg G, Kölle D, Strohmeyer D, et al. Age-related rhabdosphincter function in female urinary stress incontinence: assessment of intraurethral sonography.

J Ultrasound Med. 2004;23:631-7.

17. Appell RA. Electrical stimulation for the treatment of urinary incontinence. Urology. 1998;51:24-6.

18. Kobashi KC, Leach GE. Stress urinary incontinence. Curr Opin Urol. 1999;9:285-90.

19. Goode PS, Burgio KL, Locher JL, Roth DL, Umlauf MG, Richter HE, et al. Effect of behavioral training with or without pelvic floor electrical stimulation on stress incontinence in women: a randomized controlled trial. JAMA. 2003;290:345-52.

20. Giuseppe PG, Pace G, Vicentini C. Sexual function in women with urinary incontinence treated by pelvic floor transvaginal electrical stimulation. J Sex Med. 2011;4:702-7.

21. Krauss DJ, Lilien O. Transcutaneous electrical nerve stimulator for stress incontinence. J Urol. 1981;125:790-3.

22. Yokozuka M, Namima T, Nakagawa $H$, Ichie $M$, Handa Y. Effects and indications of sacral surface therapeutic electrical stimulation in refractory urinary incontinence. Clin Rehabil. 2004;18:899-907.

23. Yang JM, Huang WC. Discrimination of bladder disorders in female lower urinary tract symptoms on ultrasonographic cystourethrography. J Ultrasound Med. 2002;21:1249-56.

24. Nishibayashi M, Kobayashi K, Miki A, Okagaki R, Nagata I, Urashima M, et al. Bladder neck evaluation by perineal ultrasound before and after reconstructive surgery for pelvic organ prolapse. J Med Ultrason. 2009;36:193-199.

25. Kim JH, Kwon OY, Jeon HS, Hwang UJ, Gwak KT, Yoon HB, et al. Effects of transcutaneous electrical stimulation on physiological symptoms and psychological satisfaction in women with stress urinary incontinence: a preliminary study. Physi Ther Korea. 2019;26:67-75. 
26. McLean L, Varette K, Gentilcore-Saulnier E, Harvey MA, Baker K, Sauerbrei E. Pelvic floor muscle training in women with stress urinary incontinence causes hypertrophy of the urethral sphincters and reduces bladder neck mobility during coughing. Neurourol Urodyn. 2013;32:1096-102.

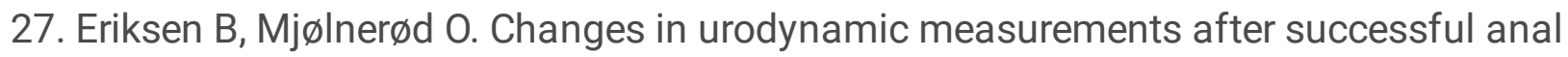
electrostimulation in female urinary incontinence. BJU Int. 1987;59:45-9.

28. Hahn I, Sommar S, Fall M. A comparative study of pelvic floor training and electrical stimulation for the treatment of genuine female stress urinary incontinence. Neurourol Urodyn. 1991;10:545-54.

29. Fall M, Lindström S. Functional electrical stimulation: physiological basis and clinical principles. Int Urogynecol J. 1994;5:296-304.

30. DeLancey JO. Structural support of the urethra as it relates to stress urinary incontinence: the hammock hypothesis. Am J Obstet Gynecol. 1994;170:1713-23.

\section{Tables}

Table 1. Stamey's grading system

\begin{tabular}{ll} 
Grade & Symptom \\
\hline 0 & No incontinence \\
\hline 1 & Incontinence on coughing, sneezing, laughing, or straining \\
\hline 2 & Incontinence on change in position or walking \\
\hline 3 & Total incontinence at all times
\end{tabular}

Table 2. Demographic characteristics of the subjects 


\begin{tabular}{ll} 
Characteristics & Total $(\mathrm{N}=21)$ \\
\hline Age $($ year $)$ & $52.24 \pm 8.11$ \\
\hline Weight $(\mathrm{kg})$ & $61.57 \pm 8.89$ \\
\hline Height $(\mathrm{cm})$ & $159 \pm 5.69$ \\
\hline Body mass index $\left(\mathrm{kg} / \mathrm{m}^{2}\right)$ & $24.24 \pm 3.21$ \\
\hline Childbirth experience $(\mathrm{n})$ & 18 \\
\hline Vaginal delivery $(\mathrm{n})$ & 13 \\
\hline Menopausal status $(\mathrm{n})$ & 13 \\
\hline Hormone drug therapy $(\mathrm{n})$ & 0 \\
\hline Onset time (year) & 7.26
\end{tabular}

Table 3. Ultrasonographic findings before and after the intervention $(\mathrm{N}=21)$ 


Before the intervention After the intervention p-value

\begin{tabular}{llll} 
& Mean \pm SD & Mean \pm SD & \\
\hline BNP at rest & $11.62 \pm 4.08$ & $12.10 \pm 4.9$ & .659 \\
\hline BNP during Valsalva maneuver & $24.38 \pm 11.32$ & $16.38 \pm 7.17$ & $.002^{\star}$ \\
\hline$\Delta$ BNP & $12.76 \pm 9.87$ & $4.29 \pm 3.89$ & $.001^{\star}$ \\
\hline LU at rest & $28.01 \pm 5.56$ & $29.68 \pm 6.19$ & .292 \\
\hline LU during Valsalva maneuver & $22.88 \pm 7.40$ & $27.43 \pm 5.64$ & $.013^{*}$ \\
\hline$\Delta$ LU & $-5.13 \pm 5.64$ & $-2.25 \pm 4.32$ & .068 \\
\hline FI at rest & $0.52 \pm .23$ & $0.51 \pm 0.45$ & .924 \\
\hline FI during Valsalva maneuver & $0.83 \pm 0.45$ & $0.54 \pm 0.45$ & $.019^{\star}$ \\
\hline$\Delta$ FI & $0.32 \pm 0.29$ & $0.23 \pm 0.25$ & $.001^{*}$ \\
\hline ART at rest & $2.89 \pm 0.91$ & $3.3 \pm 0.96$ & $.044^{*}$ \\
\hline ART during Valsalva maneuver & $2.4 \pm 0.72$ & $3.62 \pm 1.23$ & $.002^{\star}$ \\
\hline$\Delta$ ART & $-0.49 \pm 0.85$ & $0.32 \pm 1.43$ & $.031^{*}$ \\
\hline PRT at rest & $2.7 \pm 0.8$ & $3.44 \pm 1.22$ & $.014^{\star}$ \\
\hline PRT during Valsalva maneuver & $2.16 \pm 0.68$ & $3.29 \pm 0.84$ & $.001^{*}$ \\
\hline$\Delta$ PRT & $-0.53 \pm 0.83$ & $-0.15 \pm 1.19$ & .208 \\
\hline
\end{tabular}

${ }^{*} \mathrm{p}<.05$

SD: standard deviation, BNP: bladder neck position, LU: length of the urethra, FI: funneling index, ART: anterior rhabdosphincter thickness, PRT: posterior rhabdosphincter thickness

Table 4. Incontinence Quality of Life scores before and after the intervention ( $N=21)$ 
Before the intervention

Total score

Avoidance and limiting behavior score

Psychosocial impact score

Social embarrassment score

${ }^{*} \mathrm{p}<.05$

Table 5. Pearson's correlation between the Incontinence Quality of Life scores and the ultrasonographic variables

\begin{tabular}{lllll} 
& Total score & AL & PI & SE \\
\hline BNP at rest & -.029 & -.175 & -.274 & .271 \\
\hline BNP during Valsalva maneuver & -.100 & -.189 & -.289 & .154 \\
\hline$\Delta$ BNP & -.244 & -.233 & -.127 & -.221 \\
\hline LU at rest & .139 & .336 & -.019 & .062 \\
\hline LU during Valsalva maneuver & $.630^{\star}$ & .236 & $.705^{\star}$ & $.488^{*}$ \\
\hline$\Delta$ LU & -.063 & -.231 & .014 & -.031 \\
\hline FI at rest & .264 & .300 & .120 & .212 \\
\hline FI during Valsalva maneuver & .156 & .312 & .109 & .004 \\
\hline$\Delta$ FI & -.310 & .089 & -.157 & $-.515^{\star}$ \\
\hline ART at rest & -.069 & -.030 & -.059 & -.062 \\
\hline ART during Valsalva maneuver & -.225 & .039 & -.188 & -.257 \\
\hline$\triangle$ ART & .306 & .247 & .283 & .112 \\
\hline PRT at rest & .335 & $.624^{*}$ & .420 & -.012 \\
\hline PRT during Valsalva maneuver & .140 & $.488^{*}$ & .028 & .016 \\
\hline$\Delta$ PRT & -.186 & .113 & -.297 & -.176 \\
\hline
\end{tabular}

${ }^{*} \mathrm{p}<.05$ 
AL: avoidance and limiting behavior, PI: psychosocial impact, SE: social embarrassment, BNP: bladder neck position, LU: length of the urethra, Fl: funneling index, ART: anterior rhabdosphincter thickness, PRT: posterior rhabdosphincter thickness

\section{Figures}

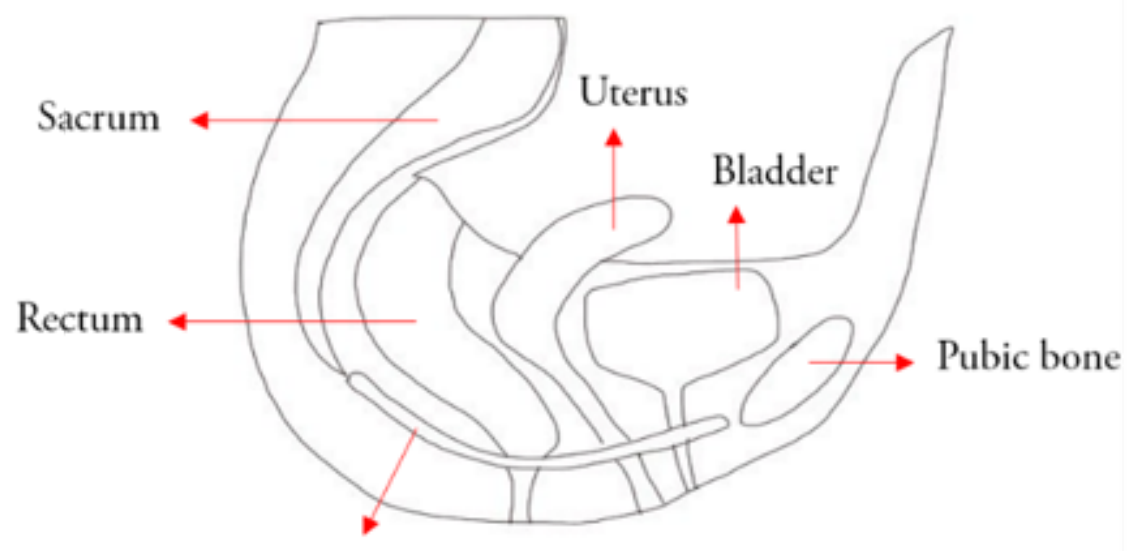

Pelvic floor

muscles

\section{Figure 1}

Mid-sagittal plane cross-section of the pelvic floor

\section{Cutaneous electrodes}

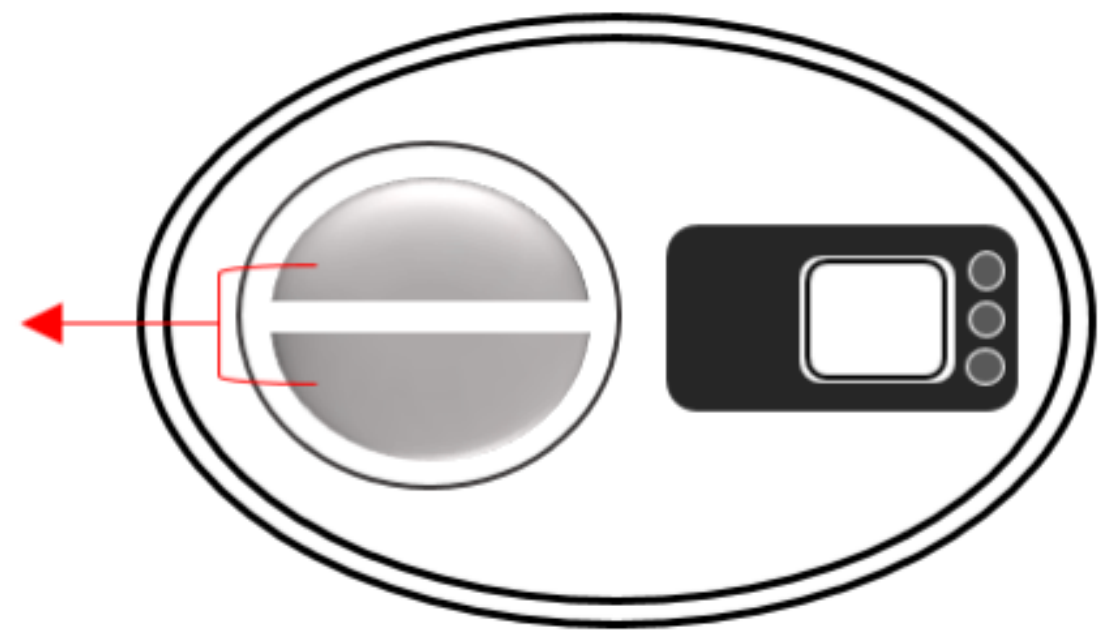

Figure 2

The transcutaneous electrical stimulation device 


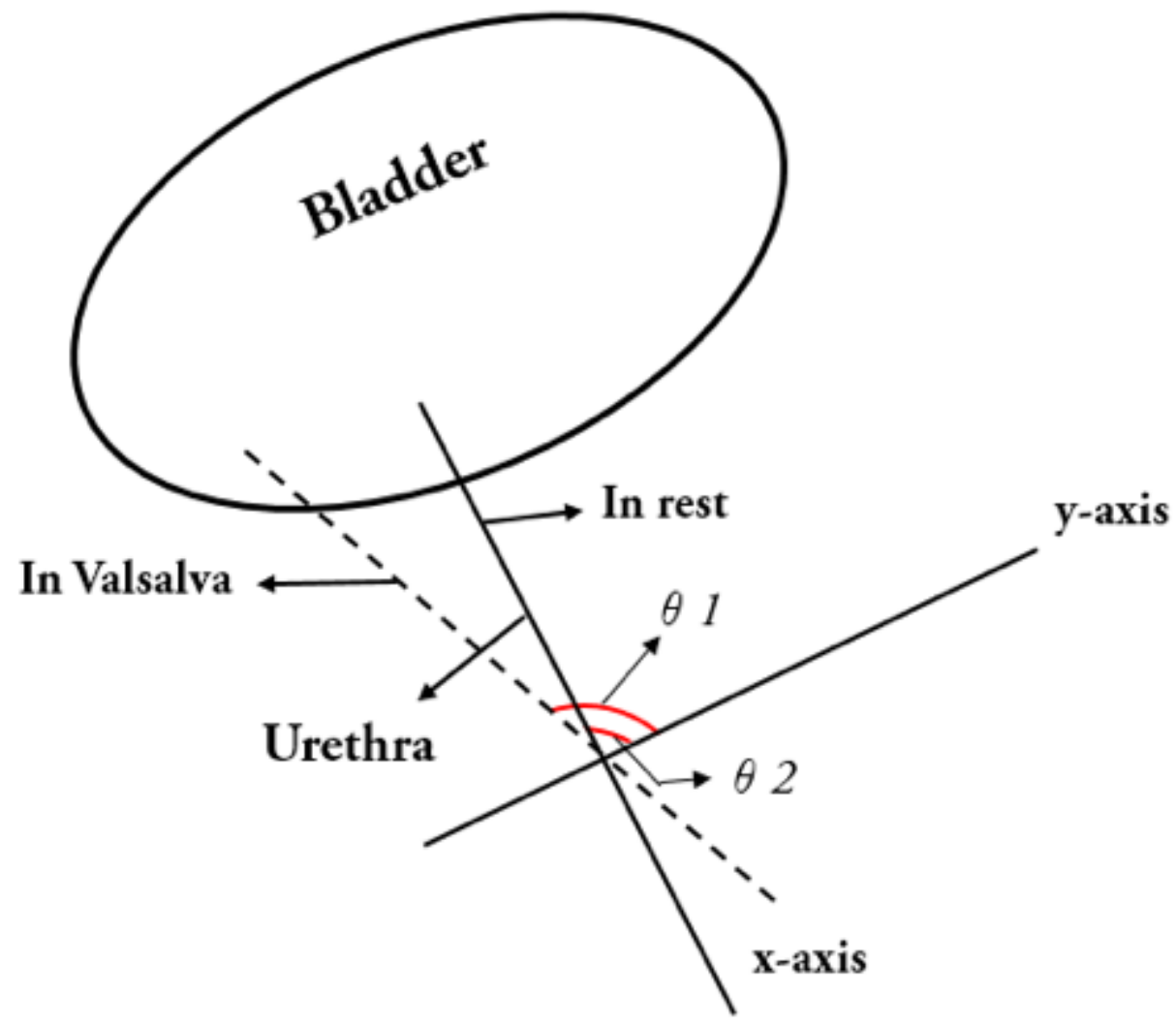

Figure 3

Schematic of an ultrasonographic view; measurement of the bladder neck position ( $\triangle$ bladder neck position $=\theta 1-\theta 2$ ) 